\title{
The effect of postmortem time, donor age and sex on the generation of neurospheres from adult human retina
}

Background/Aim: Postmortem adult human retina contains pluripotent progenitor cells capable of forming neurospheres with different retinal cell types. The authors examine whether this is the case at all ages and at different postmortem times.

Methods: Adult human postmortem retina-derived cell suspensions generated neurospheres in fibroblast growth factor 2 and N2 supplement. The yield of neurospheres from limited dilution or single cell cultures is very low so the authors studied cells generated per $10^{5}$ viable cells from a cell suspension derived from whole retina. Retinal tissue from donors aged 18-91 at various postmortem times (between 23-44 h) was studied in the context of generation rate and time for neurospheres.

Results: The potential to generate neurospheres from adult human retina remains throughout life. Neurosphere cellular components were not affected by donor age or postmortem time (they contained nestin ${ }^{+}$, glial fibrillary acidic protein ${ }^{+}$and neurofilament ${ }^{+}$cells). An average of 34.36 neurospheres were generated per $10^{5}$ viable cells. After a few days in culture neurospheres begin to form. The time for this to occur was independent of donor age but prolonged at longer postmortem times. No significant effect of donor sex was found.

Conclusion: Neurosphere-forming retinal progenitor cells are found in adult human retina throughout life. This cell population is a potential target for therapeutic intervention to influence repair and regeneration of the retina.

$\mathrm{R}$ ecovery of function in the central nervous system (retina, brain and spinal cord) is an increasingly accepted phenomenon. It is likely that an inherent regenerative capacity is present throughout life. This may occur through a number of mechanisms including cellular replacement, synaptic plasticity and architectural tissue reconformation. The recognised potential of progenitor cells to generate new cells supports this concept. Neural progenitor cells are present in the prenatal, developing and postnatal human retina. ${ }^{1-3}$ Retinal neural progenitors are present in the adult human retina in reduced numbers compared to developing tissue. ${ }^{4-7}$ Retinal neural progenitor cells generate neurospheres and differentiate into several cell types including glia, neurons and photoreceptors. ${ }^{1-6}$ The presence of these cells is potentially important for tissue repair as the retina has the framework to sustain the migration of these cells.

Cell suspensions from adult human postmortem retina generate neurospheres from cells capable of division and differentiation. ${ }^{6}$ This work not only demonstrated progenitor cells but also that they could be derived from postmortem tissue and passaged in vitro (unpublished data, ${ }^{6}$ ). We describe the potential to generate neurospheres from donors of different age and sex, as well as the effect of postmortem time.

\section{METHODS \\ Cell preparation and culture}

Donor tissue from the National Corneal Transplant Service Eyebank (University of Bristol, Bristol Eye Hospital) was obtained with research consent and ethical approval (Central and South Bristol Research and Ethics project number E5866). Eyes were obtained from donors without known concomitant ocular disease. The lens was removed with the iris; the ciliary body and pars plana were dissected, followed by removal of the vitreous. As the vitreous was removed from the eyecup, in its entirety, the often detached retina could be stripped from the hyaloid face and remained whole within the eyecup attached to the optic nerve. The whole retina was removed en bloc by dissecting it from the optic nerve head. Retinal pigment epithelium, choroid and ciliary body remained within the eyecup. This permits selective dissection of the whole retina. The retina was placed in a Petri dish to expose all areas and rinsed with Dulbecco's modified Eagle's medium (DMEM) (GIBCO, UK) to remove any retinal pigment epithelial cells that may have remained attached. Cell suspensions were prepared from whole neural retina following enzymatic digestion at $35^{\circ} \mathrm{C}$ (with trypsin, DNAse and collagenase; SIGMA, UK) and mechanical disruption (trituration with 19,21 and 23 g needles). Cell suspensions were washed and passed through a $40 \mu \mathrm{m}$ sieve to remove debris. Cells were suspended in DMEM:HAMS F12 (GIBCO) at a density of $2 \times 10^{6}$ cells/10 ml. Culture medium was supplemented with FGF-2 (SIGMA) at $20 \mathrm{ng} / \mathrm{ml}$ and $1 \%$ neural supplement (N2 supplement, GIBCO). Cell suspensions were cultured for one month and neurosphere generation was quantified. Neurospheres were confirmed by noting retinal lineage phenotype, as previously described. ${ }^{6}$

Cell suspensions generated from 50 donors ( 25 male and 25 female) were compared to generate the data presented. Donor age was 18-91 years with up to $48 \mathrm{~h}$ postmortem times. Neurospheres were counted in culture using phase-contrast microscopy. Cellular lineage was confirmed with immunocytochemistry. ${ }^{6}$

Neurospheres were counted in suspension at various time points in vitro. The number of neurospheres per $10^{5}$ vital cells was used as an index of the ability of a cell suspension to generate neurospheres ( $\mathrm{NSph} / 10^{5}$ cells). The time for neurospheres to begin to form was also recorded.

\section{Statistical analysis}

Data were analysed with Spearman correlations, ANOVA and Mann-Whitney tests, using StatView from the SAS Institute Inc (USA).

\section{RESULTS}

The generation of neurospheres from adult human retina is a consistent phenomenon; although working with postmortem

Abbreviations: CNS, central nervous system; DMEM, Dulbecco's modified Eagle's medium 
tissue generates some variability. The rate at which this occurs is recognised to be low. ${ }^{2}$ These data attempt to identify factors contributing to this variability in rates of neurosphere generation.

\section{Neurospheres generated from postmortem adult human retina}

Retinal cell suspensions were cultured in the presence of fibroblast growth factor 2 and N2 supplement for one month. Neurospheres were identified under phase-contrast as free floating balls of cells. ${ }^{6}$ Neurospheres contained: (a) nestin ${ }^{+}$cells (b) cells of various retinal lineages including glial, neuronal and photoreceptors. ${ }^{6}$ The neurospheres increased in size and number in vitro with time. In addition to cellular phenotypes previously demonstrated within spheres, BrdU incorporation and increasing cell counts showed cell numbers were increasing. ${ }^{6}$ Retinal cellular phenotypes were confirmed in these cultures.

\section{Effect of donor age on neurosphere-generating ability of retinal cell suspensions}

Our investigations have studied retina from donors aged 1891 years. Retinal cell suspensions of all donor ages generated neurospheres, ${ }^{6}$ at an average of 34.36 neurospheres per $10^{5}$ viable cells. With increasing donor age, the number of neurospheres generated per $10^{5}$ viable cells increased slightly; this correlation was found to be statistically significant (Spearman's $\mathrm{r}=0.334 ; \mathrm{p}=0.0193$ ) (fig $\mathrm{lA}$ ). The time to generate neurospheres in vitro showed no statistically significant correlation with donor age (Spearman's $\mathrm{r}=0.114 ; \mathrm{p}=0.4263$, not significant (NS), fig 1B). Thus neurospheres formed at similar time intervals in vitro regardless of donor age.
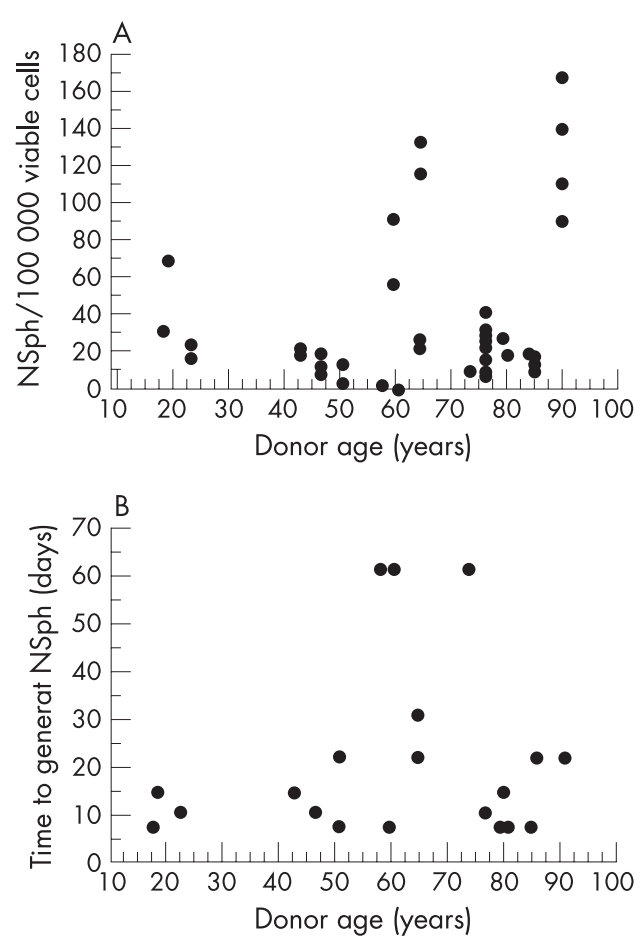

Figure 1 Donor age and ability to generate neurospheres (NSph). (A) With increasing donor age there was a slight increase in the number of neurospheres generated per $10^{5}$ viable cells (Spearman's $r=0.334$; $p=0.0193$ ). (B) donor age had no effect on the time to generate neurospheres (Spearman's $r=0.114 ; p=0.4263, N S$ ).
Effect of postmortem time on neurosphere generation Several non-human mammalian studies have found that neurospheres can be generated from the central nervous system (CNS) with prolonged postmortem times. ${ }^{8-10}$ This is possible with adult human retina. Statistical analysis of postmortem time versus the number of neurospheres generated per $10^{5}$ viable cells (fig $2 \mathrm{~A}$ ) found no statistically significant correlation (Spearman's $\mathrm{r}=-0.24 ; \mathrm{p}=0.8679$, NS). Thus although there was a slight decrease in the generation of neurospheres from tissue with longer postmortem times, this failed to reach statistical significance up to $48 \mathrm{~h}$ postmortem. There were few postmortem times below $24 \mathrm{~h}$, reflecting the time to arrange consent, transport and screening of human donor tissue. There was a statistically significant (Spearman's $r=0.597 ; \mathrm{p}<0.001$ ) increase in the time to generate neurospheres in vitro with increasing postmortem time (fig 2B). Neurospheres formed significantly more slowly in vitro from tissue with longer postmortem times.

\section{Effect of donor sex on neurosphere generation}

Analysis of variance found no statistically significant difference between the number of neurospheres generated per $10^{5}$ viable cells between male and female donors $\left(F_{1,48}=0.476 ; p=0.624\right.$, NS). In this data set, the mean time to generate neurospheres was shorter for males (mean 12.5, SEM 2.072, range 7-60 days) than females (mean 28.2, SEM 3.844, range 7-60 days) and this difference was statistically significant (ANOVA $\mathrm{F}_{1,48}=12.956 ; \quad \mathrm{p}=0.008$, significant). However, MannWhitney testing found that the mean postmortem time for male donors $(21.42 \mathrm{~h}$ ) was significantly different from that for female donors $(29.58 \mathrm{~h}) \quad(\mathrm{U}=210.5, \mathrm{Z}=-1.979, \mathrm{p}=0.048)$. Given the above described effect of postmortem time on the time for neurospheres to form in vitro, the effect of donor sex on the time to generate neurospheres can be attributed to this
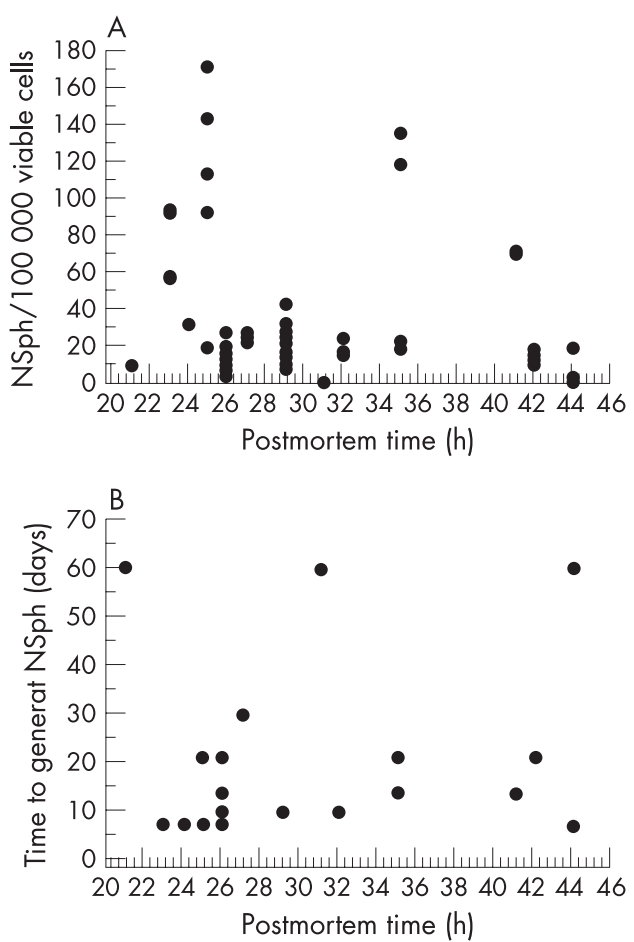

Figure 2 Postmortem time and neurosphere (NSph) generation. (A) There was no significant correlation between postmortem time and the number of neurospheres generated per $10^{5}$ viable cells (Spearman's $r=-0.24$; $p=0.8679$, NS). (B) Extended postmortem time retarded neurospheres generation (statistically significant correlation $r=0.597 ; p<0.001$, highly significant). 
confounding variable in the data (an unequal distribution of postmortem times between male and female donors).

\section{DISCUSSION}

Since the identification of progenitor cells within the adult mammalian eye $\mathrm{e}^{4-7}$ there has been much interest in identifying and characterising progenitor cells within developing and adult human retina. $^{1-7}$ We have documented the presence of progenitor cells within postmortem adult human retina and also the characteristic ability of these cells to generate neurospheres that contain retinal lineage specific cells. ${ }^{67}$ The question then arose as to whether this potential remains throughout life. The data presented highlight that adult human postmortem retina contains cells able to generate neurospheres irrespective of donor age or postmortem time (up to $48 \mathrm{~h}$ ). Neurospheres were generated from donor retinal cell suspensions irrespective of donor age (18-91 years). Donor age did not significantly affect the time (days) to generate an average of 34.36 neurospheres per $10^{5}$ viable cells of whole retina.

Several non-human studies have shown that neurospheres can be generated from the CNS with prolonged postmortem times. ${ }^{8-10}$ In rodents, postmortem time did not influence neurosphere generation from CNS progenitor cells, when the CNS tissue was stored at room temperature for up to $30 \mathrm{~h}$ or at $4^{\circ} \mathrm{C}$ for up to $48 \mathrm{~h}$. Progenitor cell phenotype and ability to differentiate was similar to cells isolated directly from healthy tissue. The data presented from postmortem human retina corroborate this; even at prolonged postmortem times, neurospheres can be passaged and differentiated cells can be generated. ${ }^{6}$

For stem cells isolated from peripheral blood, yields of viable cells are greater from male than female donors; ${ }^{11}$ our data show that the number of neurospheres generated from donor retina was not sex-dependent. Our finding that neurospheres from male donors formed more rapidly was associated with a skewed distribution of postmortem times between the male and female donors. This effect of donor sex is attributed to differences in postmortem time (confirmed as a significant determining factor in neurosphere generation).

The adult human retina generates neurospheres from single cell cultures at an extremely low rate, ${ }^{2}$ comparable with the rates which we describe. ${ }^{6}$ As a result, it is necessary to culture large numbers of cells in order to study neurosphere generation. We therefore elected to study whole retina to reduce sampling bias and increase our yield of neurospheres rather than carry out limited dilution experiments. Our major interest was whether neurospheres could be generated and at what rate per $10^{5}$ cells. To justify limited dilution experiments and single cell cultures, enriched and isolated progenitor populations are needed. When possible these experimental strategies will represent a viable experimental approach for analysing growth factor and transcriptional control of progenitor cell fate in this cell population. Understanding and facilitating progenitor cell differentiation will open possibilities of optimising tissue repair and regeneration. It is reasonable to anticipate that in order to function in their natural microenvironment, progenitor cells must respond to signals, which guide their proliferation, differentiation and integration. ${ }^{3}{ }^{12-14}$

Cells that generate neurospheres remain in the human retina throughout life. We have qualitative data to support retinal specific phenotypes in the spheres, but have not quantified variability in differentiation capacity that may exist at different ages or with extended postmortem times.

The presence of viable retinal progenitor cells throughout life in the human retina and their potential to be cultured from tissue after extended postmortem times is important. These cells are potential targets for therapeutic manipulation, to direct their behaviour in situ or for developing transplantation (experimental or therapeutic) paradigms.

\section{ACKNOWLEDGEMENTS}

We acknowledge the support of the National Eye Research Centre, the Guide Dogs for the Blind Association, the Iris Fund for the Prevention of Blindness and the Robert McAlpine Trust. Tissue was provided by the CTC National Eyebank at Bristol Eye Hospital.

\section{Authors' affiliations \\ D A Carter, E J Mayer, A D Dick, Department of Clinical Sciences South Bristol, University of Bristol, Bristol Eye Hospital, Bristol, UK}

EJ Mayer is supported by a National Career Scientist Award from the Department of Health and NHS R\&D.

Competing interests: None declared.

Correspondence to: Mr E J Mayer, Department of Clinical Sciences, University of Bristol, Bristol Eye Hospital, Lower Maudlin Street, Bristol BS1 2LX, UK; e.mayer@bristol.ac.uk

Accepted 1 April 2007

Published Online First 23 May 2007

\section{REFERENCES}

1 Klassen H, Ziaeian B, Kirov II, et al. Isolation of retinal progenitor cells from postmortem human tissue and comparison with autologous brain progenitors. J Neurosci Res, 2004;77, 334-43.

2 Coles BL, Angenieux B, Inoue T, et al. Facile isolation and the characterization of human retinal stem cells. Proc Natl Acad Sci U S A 2004;101:15772-7.

3 Yang P, Seiler MJ, Aramant RB, et al. In vitro isolation and expansion of human retinal progenitor cells. Exp Neurol 2002;177:326-31.

4 Tropepe V, Coles BL, Chiasson BJ, et al. Retinal stem cells in the adult mammalian eye. Science 2000;287:2032-6.

5 Ahmad I, Tang L, Pham H. Identification of neural progenitors in the adult mammalian eye. Biochem Biophys Res Commun 2000;270:517-21.

6 Mayer EJ, Carter DA, Ren Y, et al. Neural progenitor cells from postmortem adult human retina. Br J Ophthalmol 2005;89:102-6.

7 Mayer EJ, Hughes EH, Carter DA, et al. Nestin positive cells in adult human retina and in epiretinal membranes. Br J Ophthalmol 2003;87:1154-8.

8 Liu X, Zhu Y, Gao W. Isolation of neural stem cells from the spinal cords of low temperature preserved abortuses. J Neurosci Methods 2006;157:64-70.

$9 X_{\mathbf{u}} \mathrm{Y}$, Kimura K, Matsumoto N, et al. Isolation of neural stem cells from the forebrain of deceased early postnatal and adult rats with protracted post-mortem intervals. J Neurosci Res 2003;74:533-40.

10 Laywell ED, Kukekov VG, Steindler DA. Multipotent neurospheres can be derived from forebrain subependymal zone and spinal cord of adult mice after protracted postmortem intervals. Exp Neurol 1999;156:430-3.

11 Ings SJ, Balsa C, Leverett D, et al. Peripheral blood stem cell yield in 400 normal donors mobilised with granulocyte colony-stimulating factor (G-CSF): impact of age, sex, donor weight and type of G-CSF used. Br J Haematol 2006;134:517-25.

12 Morshead CM, Reynolds BA, Craig CG, et al. Neural stem cells in the adult mammalian forebrain: a relatively quiescent subpopulation of subependymal cells. Neuron 1994;13:1071-82.

13 Morshead CM. Adult neural stem cells: attempting to solve the identity crisis. Dev Neurosci 2004;26:93-100.

14 Goldman SA, Sim F. Neural progenitor cells of the adult brain. Novartis Found Symp 2005;265:66-80; discussion 2-97. 